Article

\title{
A Nuclear Emulsion Detector for the Muon Radiography of a Glacier Structure
}

\author{
Akitaka Ariga ${ }^{1}$, Tomoko Ariga ${ }^{1,2} \mathbb{1}^{\mathbb{D}}$, Antonio Ereditato ${ }^{1}$, Samuel Käser ${ }^{1}$, Alessandro Lechmann ${ }^{3}$, \\ David Mair ${ }^{3}$, Ryuichi Nishiyama ${ }^{1}$, Ciro Pistillo ${ }^{1}$, Paola Scampoli ${ }^{1,4}, *$ (D) , Fritz Schlunegger ${ }^{3}$ (D) \\ and Mykhailo Vladymyrov ${ }^{1}$ \\ 1 Albert Einstein Center for Fundamental Physics, Laboratory for High-Energy Physics, University of Bern, \\ Bern 3012, Switzerland; akitaka.ariga@lhep.unibe.ch (A.A.); tomoko.ariga@lhep.unibe.ch (T.A.); \\ antonio.ereditato@cern.ch (A.E.); samuel.kaeser@students.unibe.ch (S.K.); \\ ryuichi.nishiyama@lhep.unibe.ch (R.N.); ciro.pistillo@lhep.unibe.ch (C.P.); \\ mykhailo.vladymyrov@lhep.unibe.ch (M.V.) \\ 2 Faculty of Arts and Science, Kyushu University, Fukuoka 812-8582, Japan \\ 3 Institute for Geological Sciences, University of Bern, Bern 3012, Switzerland; \\ alessandro.lechmann@geo.unibe.ch (A.L.); david.mair@geo.unibe.ch (D.M.); \\ fritz.schlunegger@geo.unibe.ch (F.S.) \\ 4 Department of Physics "Ettore Pancini”, University of Naples Federico II, Napoli 80126, Italy \\ * Correspondence: paola.scampoli@lhep.unibe.ch; Tel.: +41-31-631-4965
}

Received: 10 April 2018; Accepted: 24 April 2018; Published: 30 April 2018

\begin{abstract}
Cosmic ray muons can be used to image the interior of geological sites provided that one employs detectors able to operate in the specific harsh conditions of the mountain environment. We designed and developed a detector exploiting the nuclear emulsion technique to assess the bedrock profile underneath an alpine glacier. Nuclear emulsions do not need any electric power supply or maintenance and allow for the measurement of the muon flux and direction behind a large target volume. The 3D density distribution of the material traversed by muons can then be assessed, bringing relevant information on the shape of the boundary between the glacial ice and the underlying bedrock. This new methodology in the geological field was recently tested in a campaign of measurements in the Jungfrau region of the central Swiss Alps. It was shown that the bedrock surface position can be measured with a resolution of about $5 \%$ when the traversed target is about $100 \mathrm{~m}$ thick. Characteristics and performance of the method are reported here and demonstrate that muon radiography based on emulsion detectors represents a powerful tool for the geological study of glaciers.
\end{abstract}

Keywords: muon radiography; glaciers; emulsion detectors

\section{Introduction}

The investigation of the interior of large structures, in particular those of geological nature such as volcanoes and mountains, is made possible by exploiting the high penetration power of the muon component of cosmic rays. A flux of muons of about 1 muon $/ \mathrm{cm}^{2} /$ minute hits the Earth at any angle and can penetrate up to several kilometers of rock, in the case of high energy particles. The radiography can then be accomplished by measuring the attenuation of the cosmic-muon flux, hence providing direct information on the density of the traversed material along the muon paths (density length). Combining data from several detectors located at different positions allows for obtaining a full knowledge of the density distribution inside the target material. This radiography technique was first proposed by George [1] and applied for the first time by Alvarez [2]. It recently 
benefited from several technological improvements and was successfully applied to image magmatic chambers of volcanos [3-5], and for archaeological purposes [6].

We recently proposed the specific application of the technique to the glacier environment of the Jungfrau region in the Central Swiss Alps to measure the shape of the boundary between ice and underlying bedrock. The main motivation for this study is the importance of knowing the bedrock topography that, in turn, provides hints on the erosional mechanism of the glacier and on the stability of the underlying rock, possibly affected by the glaciers' shrinkage due to the global warming. Muon radiography offers a powerful tool to map the base of glaciers.

Our first results in visualizing the bedrock topography [7] were obtained with a muon detector based on nuclear emulsion films, a technique already used for volcanoes' investigation, together with others, such as plastic scintillators [4] and resistive plate chambers [5]. The detectors had to be installed below the bedrock level, which was possible thanks to the existence of a railway tunnel underneath the target. This circumstance also allowed the installation of the detectors at the base of the glacier from several perspectives, such to cover several directions of the incoming muons.

This paper focuses on the detector aspects of the study and on the related optimization of the emulsion technique. In particular, the incident muon flux and direction have to be measured with high angular resolution and, at the same time, the detector must be easily portable, possibly devoid of electric power, and requiring minimal maintenance. Nuclear emulsion is a very old technique [8,9], recently revived thanks to the achievement of a fast automatic scanning of particle tracks [10]. This is a key issue for the analysis of large area detectors needed to reach an adequate spatial resolution in mapping the target. Moreover, we had the opportunity offered by the scanning facility at the University of Bern, the largest in Europe, which is comprised of six microscopes for the automatic scanning of the detectors [11,12].

\section{3D Cosmic Muon Radiography Applied to Alpine Glaciers}

As mentioned above, muon tomography relies on the attenuation of cosmic muons through the target material as a function of angles and positions. This attenuation rate depends on the density path traversed by muons (density-length) described as:

$$
X=\int_{L} \rho(s) d s=L\langle\rho\rangle,
$$

where $\langle\rho\rangle$ is the averaged density along the trajectory with length $L$. The density length can then be obtained from the attenuation of the muon flux. In the event that the structure to be imaged is made of two different materials with known densities $\rho_{1}$ and $\rho_{2}$, and lengths $L_{1}$ and $L_{2}$, and we know the total length $L$ (see Figure 1), then $L_{1}$ and $L_{2}$ can be assessed through $L=L_{1}+L_{2}$ and $X=\rho_{1} \cdot L_{1}+\rho_{2} \cdot L_{2}$, where $X$ can be inferred from the muon flux measurement. Observations can be performed at different orientations with respect to the object one wants to image. The combination or merger of the different density lengths allows for obtaining a computed axial tomography. For a review of the methodology, see, for example, [13,14].

Muon radiography requires the knowledge of the incident muon energy spectrum at the site of the measurement, as, for many, geological applications its direct measurement is unfeasible, one has to rely on models benchmarked by experimental data. Muons are produced from the decay of charged mesons at altitudes of typically $15 \mathrm{~km}$. Their energy spectrum and angular distribution are a convolution of the production spectrum, energy loss, and decay point in the atmosphere. Figure 2 (left) shows the energy spectrum at sea level for different zenith angles. The spectrum is almost flat below $10 \mathrm{GeV}$. It gradually steepens in the $10-100 \mathrm{GeV}$ range, thereby reflecting the primary spectrum, and further steepens at higher energies.

The muon energy spectrum was throughly studied both experimentally and theoretically. One of the most successful models was proposed by Reyna et al. [15]; this well represents the data from experiments and it has been widely employed for previous muon radiography studies [16]. The muon 
spectrum at the sea level $\Phi(p, \theta, h=0 \mathrm{~m})$ as a function of momentum $p(\mathrm{in} \mathrm{GeV} / \mathrm{c})$ and zenith angle $\theta$ is given by:

$$
\begin{aligned}
\Phi(p, \theta, 0) & =\cos ^{3} \theta \Phi_{\mathrm{V}}(p \cos \theta), \\
\Phi_{\mathrm{V}}(\zeta) & =c_{1} \zeta^{-\left(c_{2}+c_{3} \log _{10} \zeta+c_{4}\left(\log _{10} \zeta\right)^{2}+c_{5}\left(\log _{10} \zeta\right)^{3}\right),} \\
c_{1} & =0.00253 \mathrm{~cm}^{-2} \mathrm{~s}^{-1} \mathrm{sr}^{-1}\left(\mathrm{GeV} / \mathrm{c}^{-1}\right), \\
c_{2} & =0.2455, c_{3}=1.288, c_{4}=-0.2555, c_{5}=0.0209,
\end{aligned}
$$

where $\zeta=(p \cos \theta)$. The altitude dependence can be described as $\Phi(p, \theta, h)=\Phi(p, \theta, 0) \exp (h / L)$, where $L=4900 \mathrm{~m}+750 \mathrm{~m} \times p($ in $\mathrm{GeV} / \mathrm{c})$ [17]. This formula is only valid up to $1000 \mathrm{~m}$ above sea level. Since our observations were performed at high altitudes $(\sim 3500 \mathrm{~m})$, we adapted this formula to $L=3400 \mathrm{~m}+1100 \mathrm{~m} \times p($ in $\mathrm{GeV} / \mathrm{c}) \cos \theta$. This adjustment was obtained by fitting the results of the muon energy spectrum at different altitudes by using a Monte Carlo air shower simulation [18].

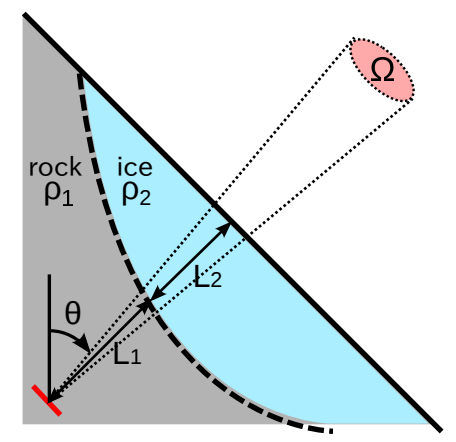

Figure 1. Schematic view of muon radiography at glacier environments.

The flux of vertical muons is $\sim 70 \mathrm{~m}^{-2} \mathrm{~s}^{-1} \mathrm{sr}^{-1}$ at sea level and decreases exponentially with the thickness of traversed material. Figure 2 (right) shows the attenuation curve for the "standard $\operatorname{rock}^{\prime \prime}$, a virtual material $\left(\mathrm{Z}=11, \mathrm{~A}=22, \rho=2.65 \mathrm{~g} / \mathrm{cm}^{3}\right)$ commonly used to discuss the underground muon flux , while the overburden material is measured in unit of "meter water equivalent" (m.w.e.). The curve is obtained by integrating the momentum spectrum (Equation (1)) for momenta higher than what is required to penetrate a given thickness of standard rock [19].
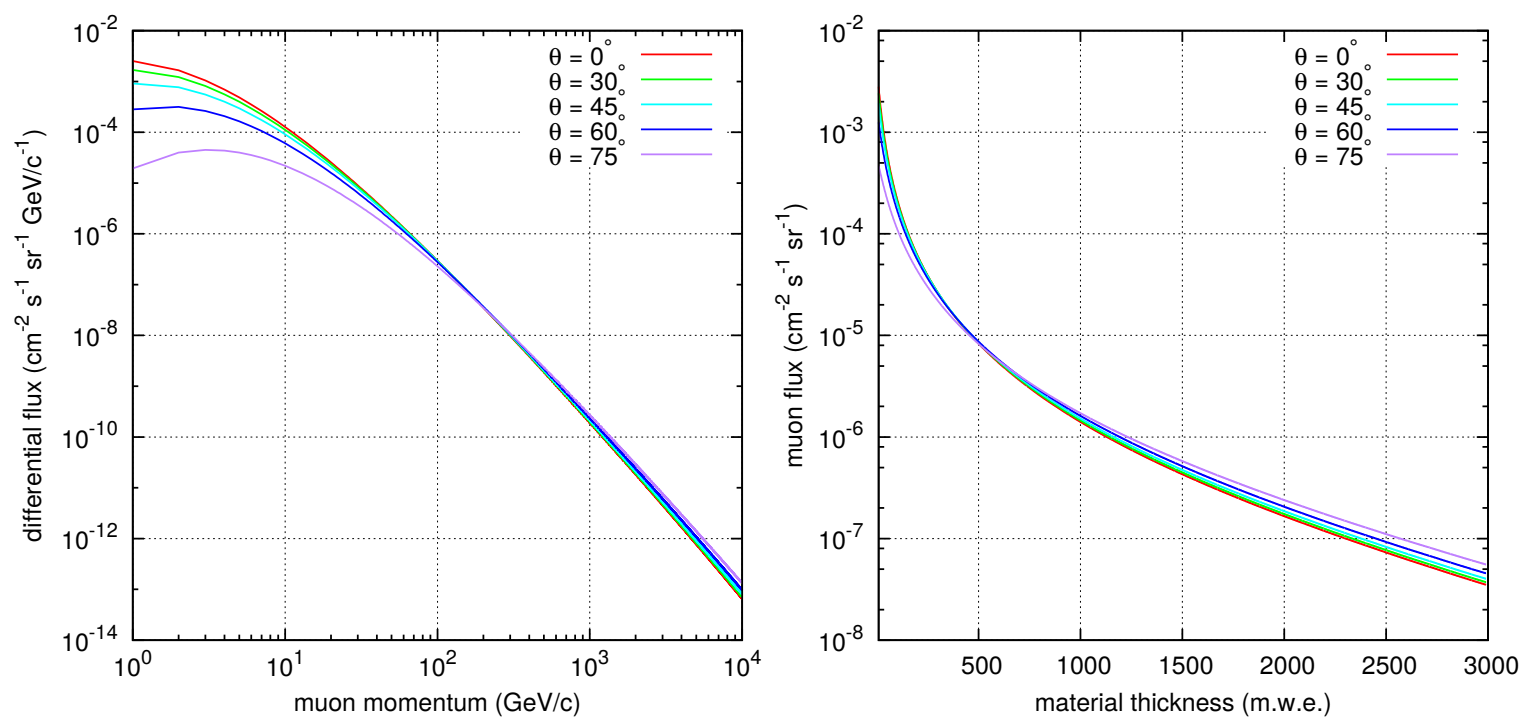

Figure 2. Energy spectrum of cosmic-ray muons for several zenith angles (left). Attenuation curve of the muon flux after passing through the "standard rock" (right). 


\section{Muon Flux Detection}

\subsection{Emulsion Film Detectors}

Distinctive features of emulsion detectors are their excellent spatial and angular resolution, and their passive nature not implying the use of electric power. Furthermore, we could also profit from the established facility at the University of Bern for automatic microscope scanning, crucial for large area detector analysis [11]. The emulsion gel consists of small silver bromide crystals dispersed in a gelatin frame. The most peculiar characteristics of the emulsion gel compared to other photographic emulsions is that the crystal size is extremely small $(0.2 \mu \mathrm{m})[8,9]$. This allows for tracing a particle trajectory with unprecedented high spatial resolution $(0.5 \mu \mathrm{m})$. Specifically, when a charged particle passes through the gel, the crystals along its trajectory create latent image centers, which become visible at the optical microscope after achemical development. The number of grains along the track of a minimum ionizing particle (MIP) quantifies the sensitivity of the detector in terms of grain density (GD), namely the number of grains $/ 100 \mu \mathrm{m}$. The typical value for GD is 30 [10], but, with special types of gels, it can reach the value of 50 [20]. The "fog" is instead the parameters representing the noise of the detector, which is due to thermally generated grains. Their volumetric density, the so called fog density (FD), typically ranges between 1 and 10 grains $/ 1000 \mu \mathrm{m}^{3}$ depending on the time elapsed from the film production. A value of FD $<3$ is considered acceptable for a noise-free analysis. Figure 3 shows the microscopic view of a chemically developed film with several tracks, whose darkness is correlated with the energy deposition of the incoming particles [21]. Since cosmic-ray muons are mostly relativistic, their tracks are seen as thin and disconnected sequences of grains.

The base unit of our detector is a plastic base film, on both sides of which the emulsion gel is poured (Figure 4). Double-side coated films have a twofold advantage: first, they allow for a precise measurement of the track angle by connecting the closest grains to the base, which are essentially not affected by distortions due to possible mechanical alterations of the detector. Secondly, they enable to take coincidences of track segments on both layers to extract the information of high energy particles including cosmic muons. Since environmental alpha or beta particles cannot penetrate the plastic base, one can easily reject them in the data analysis. Track segments reconstructed in a single emulsion layer are called "micro-tracks". If two micro-tracks on different layers are straightly aligned, one can build the so-called "base-tracks". Micro-tracks and base-tracks are automatically reconstructed by a readout scanning system developed on purpose (Section 4). The thickness of base and emulsion layers can be adjusted according to the specific goal; for our purposes, they are $200 \mu \mathrm{m}$ and $50 \mu \mathrm{m}$, respectively.

The emulsion gel was produced at Nagoya University, while the emulsion films were manufactured at the underground facility of the University of Bern. This comprises nine stainless tables for pouring the gel on the plastic bases. The viscosity of the gel can be precisely controlled by varying the temperature of the tables. This facility allows for producing about $10 \mathrm{~m}^{2}$ of films per week.

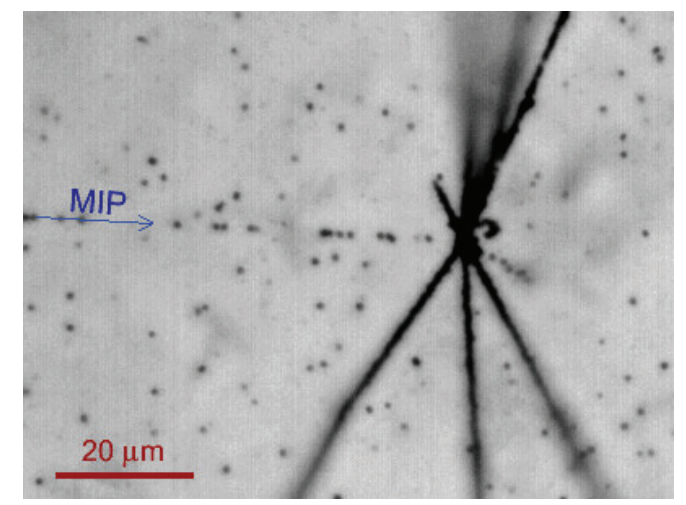

Figure 3. Microscopic view of the emulsion films with several types of tracks (from [21]). The thicker the tracks, the higher is their energy loss. 


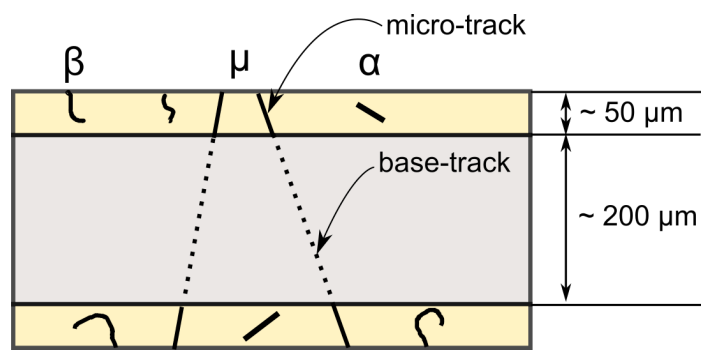

Figure 4. Schematic view of emulsion film. Definition of micro-track and base-track.

\subsection{Detector Design}

The aim of the measurement is to estimate the ice depth $x\left(L_{2}\right.$ in Figure 1) by means of the muon flux attenuation analysis to assess the glacier bedrock profile. The expected flux at the detector varies strongly with $x$ because of the large density difference between ice and bedrock density [22]. Figure 5 shows the muon flux expected for several ice thicknesses $(x)$ for a total target thickness $L$ of $100 \mathrm{~m}$ and a zenith angle $\theta$ of $45^{\circ}$. The resolution in the measurement of the bedrock position can be defined as the minimum difference of the ice depth $(\Delta x)$ whose influence on the flux would be significant enough compared to the uncertainty in the flux measurement $(\Delta \Phi)$. The error on the flux originates from fluctuations of the number of detected muons, governed by Poisson statistics. When occurrences are large enough $(\geq 10)$, their fluctuations can be approximated by a Gaussian distribution with mean value $N_{\mu}$ and standard deviation $\sqrt{N_{\mu}}$. Therefore, the flux uncertainty decreases with increasing $N_{\mu}$ $\left(\Delta \Phi / \Phi=\Delta N_{\mu} / N_{\mu}=1 / \sqrt{N_{\mu}}\right)$ and hence improves with increasing detector size (S) times exposure time (T). Figure 5 (right) represents the expected resolution $(\Delta x)$ as a function of $S \times T$ when the solid angle is $\Omega=0.01 \mathrm{sr}$. To achieve a resolution of $\Delta x=5 \mathrm{~m}$ at the $99.7 \%$ confidence level, $S \times T$ must be at least $10^{9} \mathrm{~cm}^{2} \mathrm{~s}$. From the above considerations, the measurement campaign required a detector surface of $S=250 \mathrm{~cm}^{2}$ and an exposure time $T=4 \times 10^{6} \mathrm{~s}$ (1.5 month). To match these needs, we designed a modular detector placed at a depth of $100 \mathrm{~m}$ below the Earth surface. Each module consisted of a stack of eight films interleaved with metal plates (lead or stainless steel, $1 \mathrm{~mm}$ thick), acting as absorbers and scatterers for low-energy particle background, like electromagnetic showers and upward-going charged particles $[5,18,23]$. Two such modules were placed in a frame $\left(12.5 \times 10 \mathrm{~cm}^{2}\right)$ yielding a $250 \mathrm{~cm}^{2}$ effective detection area.
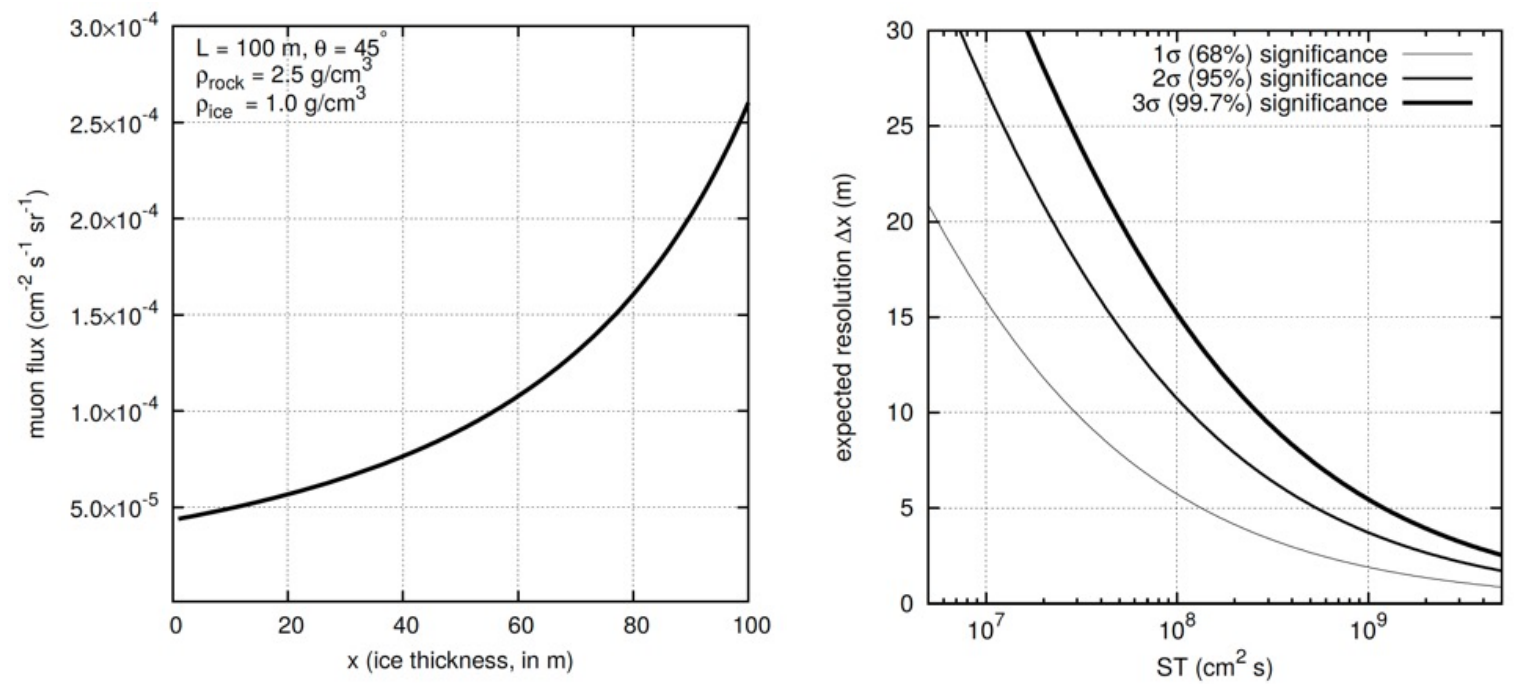

Figure 5. Expected muon flux as a function of the ice thickness $x$ for the reference case of $\rho_{\text {ice }}=1.0 \mathrm{~g} / \mathrm{cm}^{3}$ and $\rho_{\text {rock }}=2.5 \mathrm{~g} / \mathrm{cm}^{3}$ (left). Expected resolution for rock thickness $\Delta x$ as a function of detector size times exposure time $T$ for a solid angle $\Omega=0.01 \mathrm{sr}$ (right). 
Figure 6 (left) shows the detector frame mounted onto the rock wall by means of a joint enabling on-site adjustments of its orientation toward the target location with an accuracy of $0.2^{\circ}$. This was accomplished thanks to reference points in the railway tunnel. Three detector frames (D1, D2 and D3) were installed about $100 \mathrm{~m}$ apart. The cross-sectional view of the uppermost detector D3 is shown in Figure 6 (right). The exposure lasted from the 16 February to the 4 April 2016 (47 days). Environmental radioactivity was shielded by thick stainless steel plates $(15 \mathrm{~mm})$ covering the modules, also ensuring the planarity of the films. After exposure, films within the same module were immediately separated to avoid further track recording during transportation, which would have seriously affected data purity. Chemical development of the films takes place at the University of Bern.
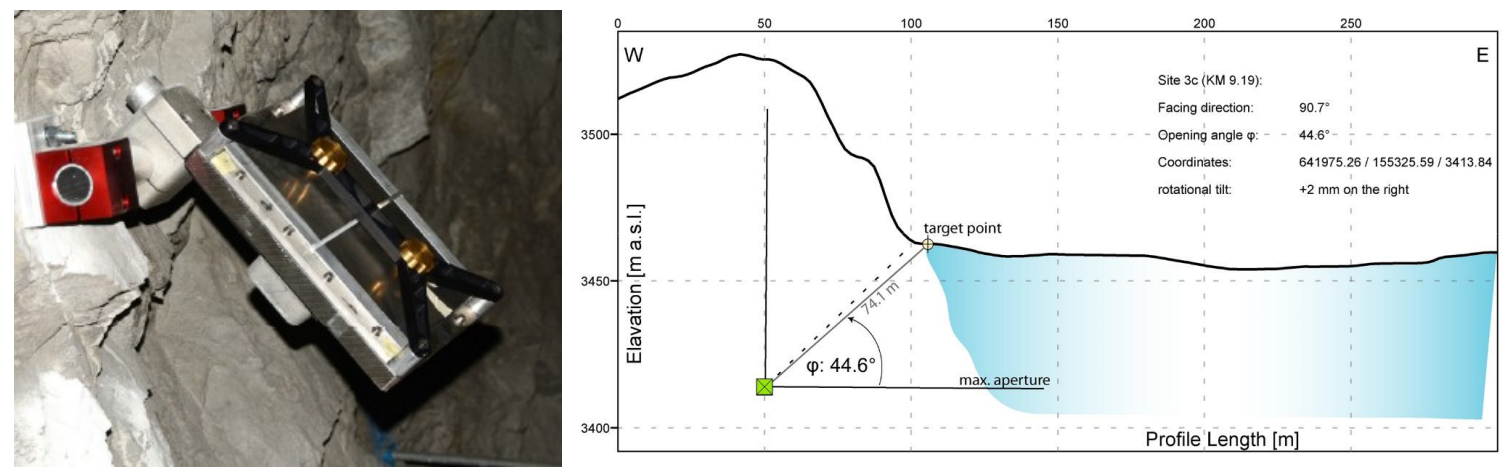

Figure 6. Detector frames installed at the rock wall in the railway tunnel (left). Cross-sectional topography with the detector D3 shown in green (right).

\section{Readout and Track Reconstruction}

All analytical and technical steps to reconstruct particle tracks in a full module are schematically summarized in Figure 7.

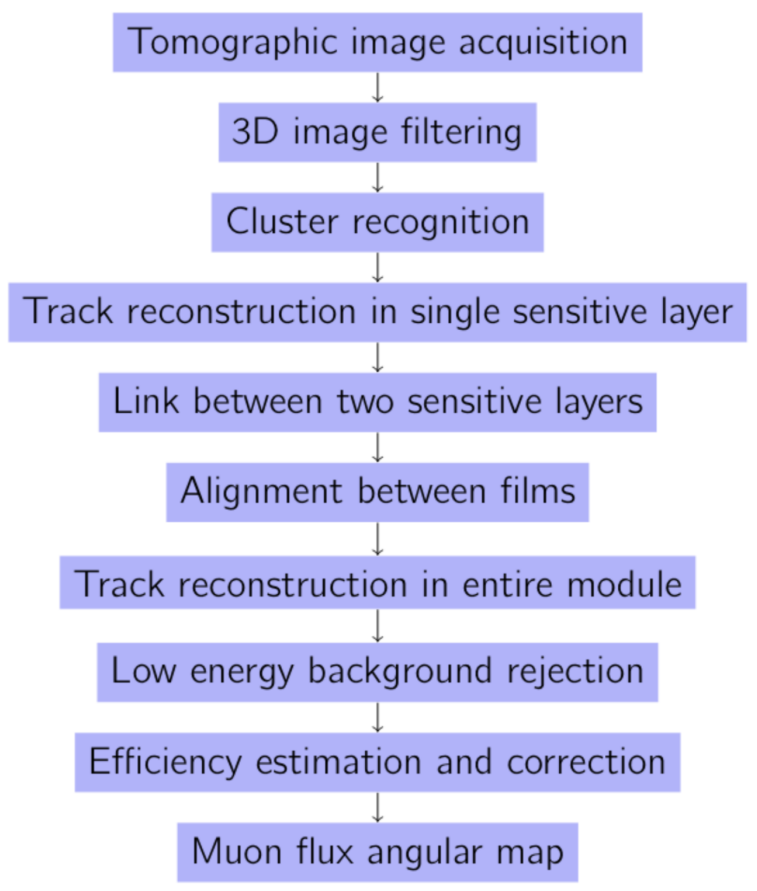

Figure 7. Procedure for the muon flux measurement. 
For each emulsion layer, the information on the muon trajectories is acquired by automated bright-field scanning microscopes. Figure 8 shows the facility at the University of Bern. The six microscopes are equipped with oil objective lenses (50× magnification), a high speed camera (1.3 Mpixel $\times 450$ FPS), and a high precision $X Y Z$ mechanical stage with a readout precision of $0.1 \mu \mathrm{m}$ in the $X-Y$ plane and $0.05 \mu \mathrm{m}$ along the $Z$-vertical axis.

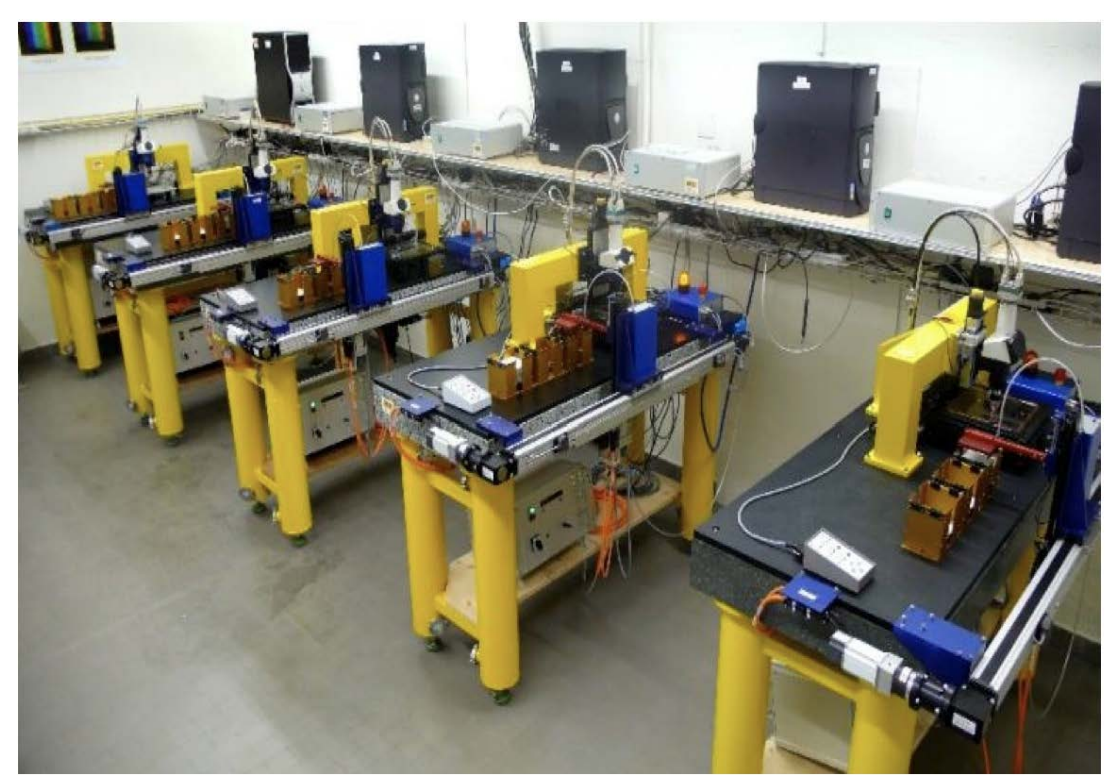

Figure 8. The six automated scanning microscopes of the University of Bern equipped with automated film exchangers. They feature a total scanning speed up to $180 \mathrm{~cm}^{2}$ emulsion surface per hour.

The microscope hardware was designed and built in house using commercial off shelf components. Tomographic images are taken for each of the two sensitive layers of the emulsion films with a field of view of $370 \mu \mathrm{m} \times 290 \mu \mathrm{m}$ and an acquisition voxel size of $0.3 \mu \mathrm{m} \times 0.3 \mu \mathrm{m} \times 1.5 \mu \mathrm{m}$. The scanning of a large emulsion film surface is performed by combining consecutive tomographic images of adjacent fields of view. An example of 3D data is shown in Figure 9.

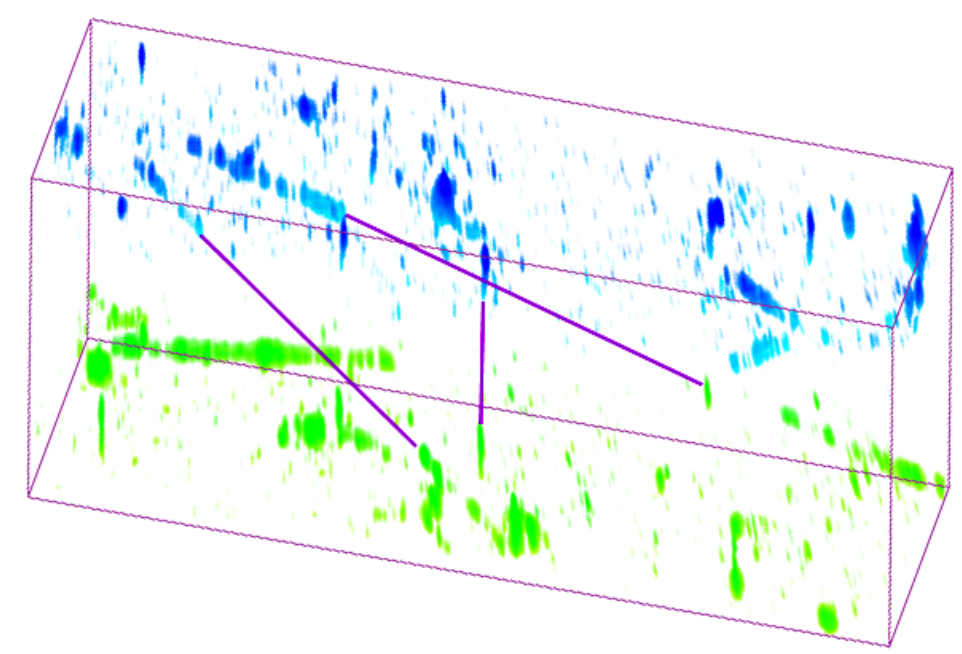

Figure 9. Tomographic data acquired by the microscope for a small volume of $150 \mu \mathrm{m} \times 30 \mu \mathrm{m} \times 300 \mu \mathrm{m}$. Blue and green colors refer to the top and bottom of sensitive layers, respectively. Purple lines represent correlated tracks between the sensitive layers (base-tracks). 
The typical raw data size for a $20 \times 25 \mathrm{~cm}^{2}$ film is 100 Tbytes. Therefore, a fast real-time data processing is crucial to collect the required data from the detectors within an acceptable time span. For this purpose, we developed a software package for high-speed image acquisition processing and micro-track reconstruction, exploiting Graphical Processors Units (GPU). The algorithms are based on the system described in [12] with some improvements principally concerning data storage and large area scanning pipeline. The typical acquisition sequence is briefly described in the following. As a first step, in order to enhance the signal to noise ratio, an image filter is applied to each field of view at different $Z$ positions. The grains are then identified in the volume by assigning to each of them the geometrical shape parameters, the spatial coordinates, and the integral brightness. Successively, the particle trajectories are reconstructed for each sensitive layer of the emulsion film by means of a linear fit to the grain coordinates (micro-tracks). This acquisition pipeline is conceptually illustrated in Figure 10. The reconstructed track segment is characterized by the three position coordinates $(x, y, z)$, the two angles $\left(\theta_{x}, \theta_{y}\right)$, the number of grain clusters and the goodness of the fit. Finally, micro-tracks in the two layers are connected. These base-tracks are classified by the $\chi^{2}$-score defined as the angular differences between each micro-track and the base-track. This $\chi^{2}$ procedure allows for rejecting background tracks such as those generated by $\beta$ rays and/or by randomly formed grains (Figure 11).
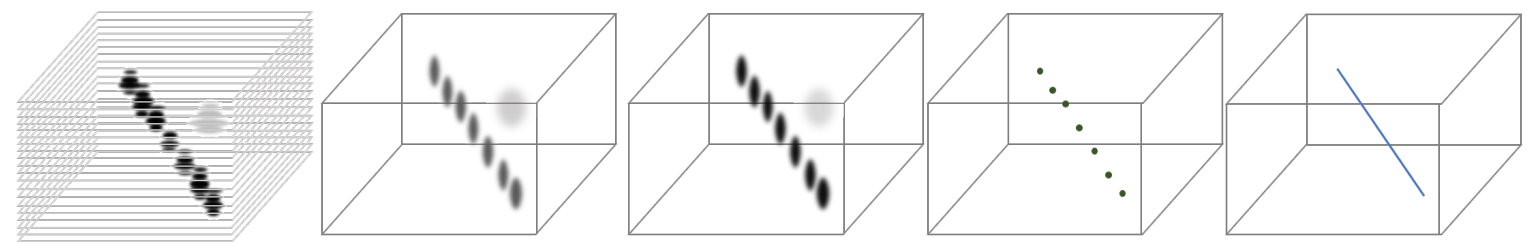

Figure 10. Schematic view of the track reconstruction sequence in a single sensitive layer of the emulsion film. From left to right: raw tomographic image stack obtained at the automated microscope; $3 \mathrm{D}$ voxels view; $3 \mathrm{D}$ voxels view after $3 \mathrm{D}$ filtration to enhance signal to background ratio; silver grains located in 3D space; reconstructed track from the grain position.

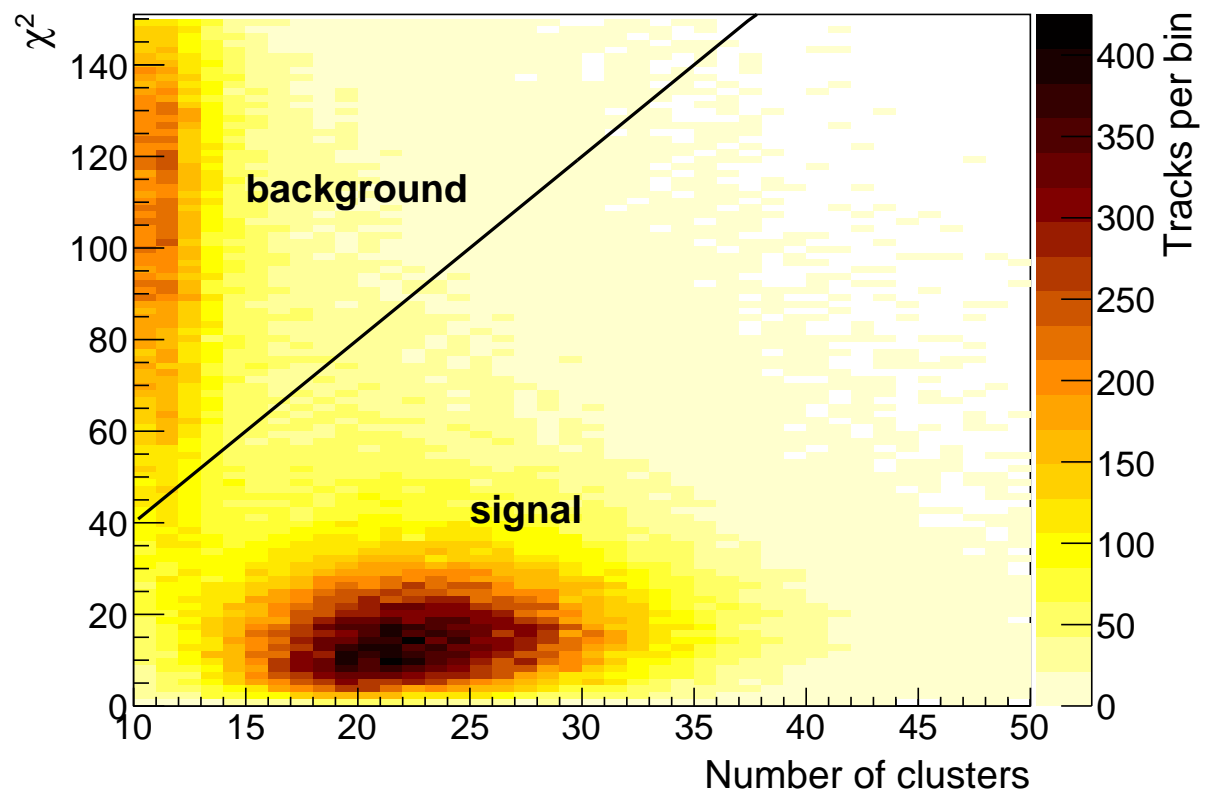

Figure 11. $\chi^{2}$ test to separate the muon signal from the background tracks ( $\beta$ rays or randomly formed grains). 
The further steps needed to reconstruct the muon tracks for an entire module are performed with the FEDRA software package (Framework for Emulsion Data Reconstruction and Analysis [24]). First, the information on the displacement between adjacent films is recovered using the tracks themselves. After this alignment process, track reconstruction is performed starting from the base tracks in the first film. Connectable base-tracks are then searched for in the following films. Reconstructed tracks are subsequently selected on the base of the number of the detected base-tracks $\left(n_{\text {base }}\right)$. In fact, those tracks characterized by a low number of base tracks $\left(n_{\text {base }}<3\right)$ are either accidental coincidence of noise signals or low energy track that stopped in the middle of the module. In addition, low energy tracks significantly bending by more than 3 mrad are also discarded since they are below the detector angular resolution. An example of some selected tracks is displayed in Figure 12a while their directional information is reported in Figure 12b.

(a)
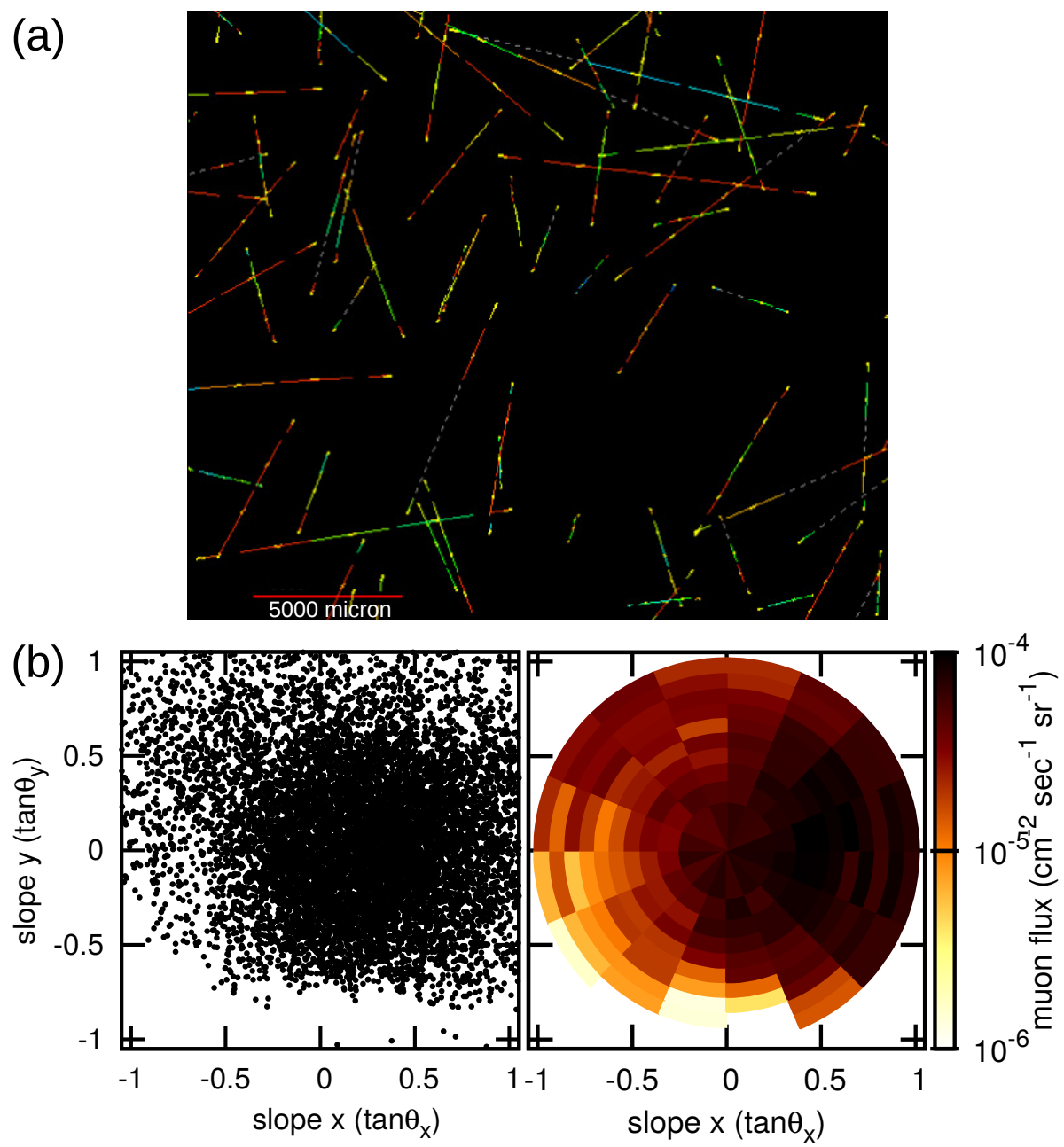

Figure 12. (a) muon tracks reconstructed in a module. The yellow segments represent the base-tracks; (b) muon angular distribution (left) and flux obtained with the detector D3 (right).

The muon flux is derived for each bin of the polar coordinate system after normalizing the number of muons for the detector size, the solid angle, the exposure time and the detection efficiency. The latter is defined for each film in a module as the probability of finding a base track at the expected position (Figure 13). It typically ranges between $80 \%$ and $95 \%$, except for nearly vertical tracks. For these tracks, the tomographic scanning procedure sees grains not well separated. Therefore, they can appear merged together and consequently their detection worsens. The reconstruction efficiency is almost $100 \%$ for the whole angular range. 


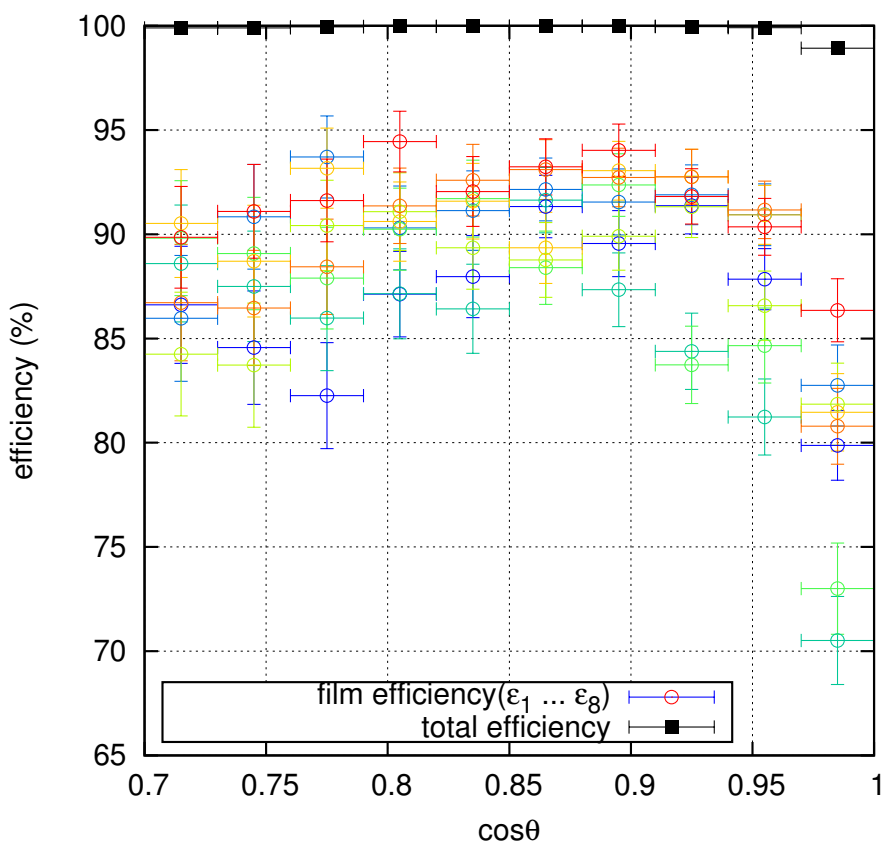

Figure 13. Base-track efficiency for each film in a module as a function of the track angle (open circles); different colors account for the eight films in a module. Track reconstruction efficiency (black squares).

\section{Results}

Figure 14 shows the observed muon flux corresponding to the pure rocky part of the mountain. The flux clearly shows the expected anti-correlation with the rock thickness. This measurement corresponds to the simulated flux obtained with a rock density of $\rho=2.68 \pm 0.04 \mathrm{~g} / \mathrm{cm}^{3}(1 \sigma)$. This value is in agreement with the measurement performed in the vicinity of the detectors installed in the railway tunnel and at the mountain surface $\left(2.65-2.74 \mathrm{~g} / \mathrm{cm}^{3}\right)$.

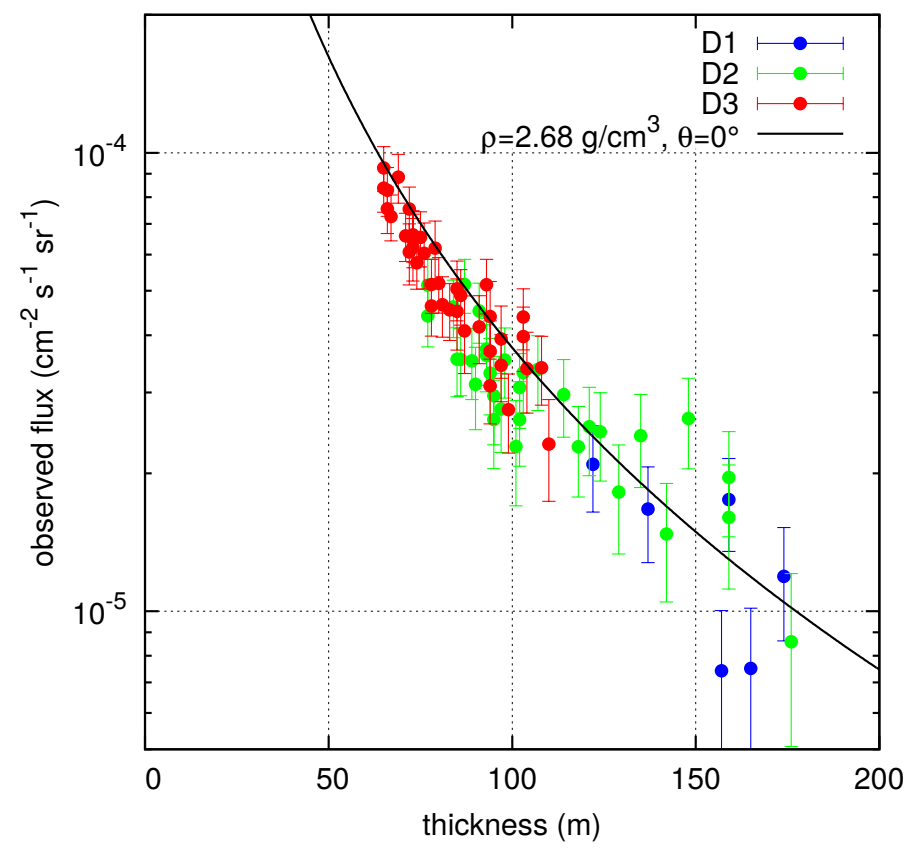

Figure 14. Muon flux measured behind the purely rocky part of the mountain as a function of the rock thickness (D1: blue, D2: green, D3: red). The theoretical attenuation for a rock density of $2.68 \mathrm{~g} / \mathrm{cm}^{3}$ and zenith angle $\theta=0^{\circ}$ is displayed as a solid curve. 
Finally, the position of the ice/rock interface is estimated as the best average density $\langle\rho\rangle$ for each bin. Assuming a bedrock density of $2.68 \mathrm{~g} / \mathrm{cm}^{3}$ and a realistic ice density of $0.80 \mathrm{~g} / \mathrm{cm}^{3}$ [22], the fraction of rock $x$ along the muon trajectory is determined as:

$$
<\rho>=\rho_{\text {rock }} \times x+\rho_{\text {ice }} \times(1-x) .
$$

The boundary position can be plotted for a distance $L x$ from the detector position as reported in [7]. Figure 15 illustrates the determined bedrock shape. On the left figure, one can see the experimental points with their uncertainty range, from $\sim 5 \mathrm{~m}(1 \sigma)$ to $\sim 20 \mathrm{~m}$. These points were used to visualize the surface of the boundary between rock and ice (Figure15, right) by properly smoothing them taking their statistical errors into account.
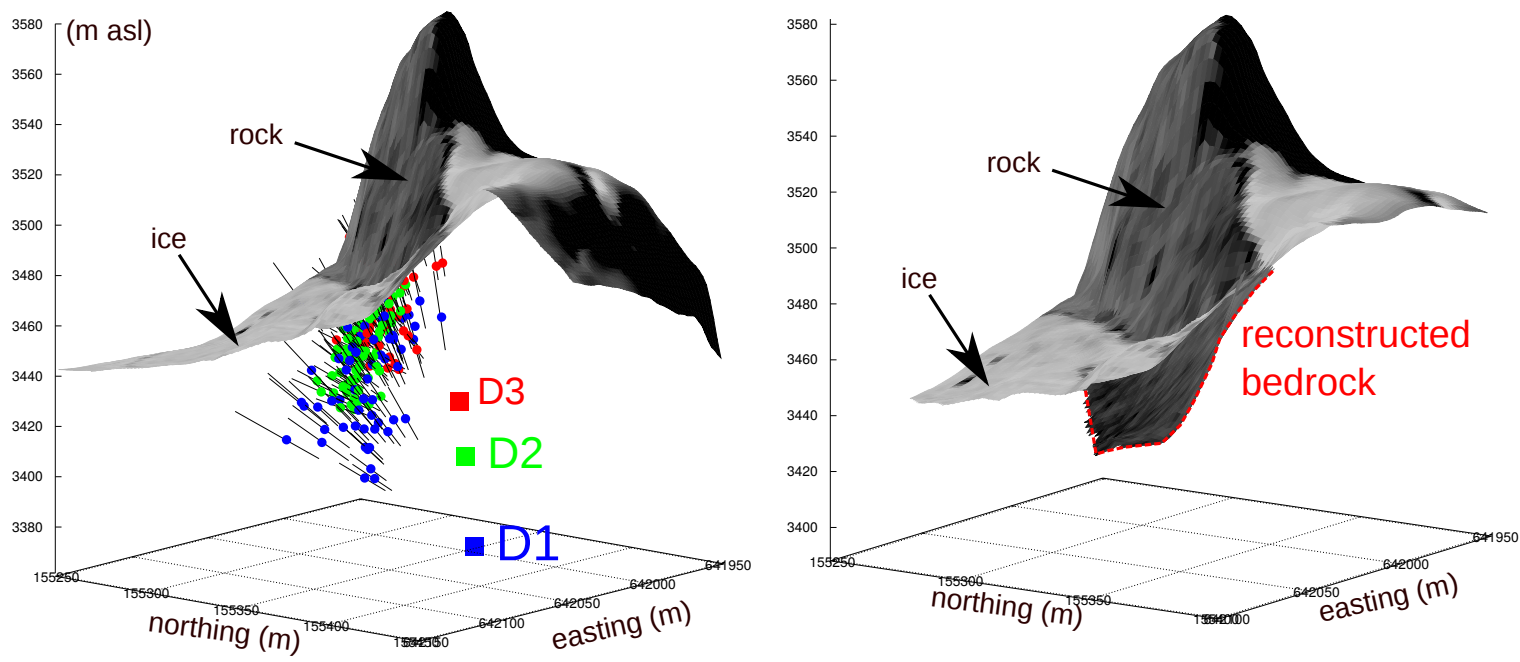

Figure 15. left: three-dimensional representation of ice/bedrock interface determined from each bin of muon flux (D1: blue, D2: green, D3: red). The error bars show the statistical uncertainty (1 $\sigma)$. right: reconstructed shape of the bedrock under the glacier. The vertical scale is magnified two times.

\section{Conclusions}

We presented here a novel application of the cosmic muon radiography technique based on emulsion film detectors, for the first time applied to the field of glaciology. In particular, we focused on the methodology, the design and the performance of the detectors. This technique provides important information when other geological procedures such as radar and seismic inspections cannot be used. In particular, we demonstrated the potentiality of emulsion detectors for the measurement of the bedrock of an Alpine glacier possibly bearing information about the erosional mechanism and on the stability of the underlying bedrock.

Acknowledgments: This project is financially supported by the Swiss National Science Foundation as an interdisciplinary research project (159299).

Conflicts of Interest: The authors declare no conflict of interest.

\section{References}

1. George, E.P. Cosmic Rays Measure Overburden of Tunnel. Commonw. Eng. 1955, 1955, 455-457.

2. Alvarez, L.W.; Anderson, J.A.; El Bedwei, F.; Burkhard, J.; Fakhry, A.; Girgis, A.; Goneid, A.; Hassan, F.; Iverson, D.; Lynch, G.; et al. Search for Hidden Chambers in the Pyramids. Science 1970, 167, 832-839. [CrossRef] [PubMed] 
3. Tanaka, H.K.M.; Nakano, T.; Takahashi, S.; Yoshida, J.; Takeo, M.; Oikawa, J.; Ohminato, T.; Aokia, Y.; Koyama, E.; Tsuji, H.; et al. High resolution imaging in the inhomogeneous crust with cosmic-ray muon radiography: The density structure below the volcanic crater floor of Mt. Asama, Japan. Earth Planet. Sci. Lett. 2007, 263, 104-113. [CrossRef]

4. Lesparre, N.; Gibert, D.; Marteau, J.; Komorowski, J.C.; Niccolin, F.; Coutant, O. Density muon radiography of La Soufrière of Guadeloupe volcano: Comparison with geological, electrical resistivity and gravity data. Geophys. J. Int. 2012, 190, 1008-1019. [CrossRef]

5. Ambrosino, F.; Anastasio, A.; Bross, A.; Béné, S.; Boivin, P.; Bonechi, L.; Cârloganu, C.; Ciaranfi, R.; Cimmino, L.; Combaret, C.; et al. Joint measurement of the atmospheric muon flux through the Puy de Dôme volcano with plastic scintillators and Resistive Plate Chambers detectors. J. Geophys. Res. 2015, 120, 7290-7307. [CrossRef]

6. Morishima, K.; Kuno, M.; Nishio, A.; Kitagawa, N.; Manabe, Y.; Moto, M.; Takasaki, F.; Fujii, H.; Satoh, K.; Kodama, H.; et al. Discovery of a big void in Khufu's pyramid by observation of cosmic-ray muons. Nature 2017, 552, 386-390. [CrossRef] [PubMed]

7. Nishiyama, R.; Ariga, A.; Ariga, T.; Käser, S.; Lechmann, A.; Mair, D.; Scampoli, P.; Vladymyrov, M.; Ereditato, A.; Schlunegger, F. First measurement of ice bedrock interface of alpine glacier by cosmic muon radiography (Aletsch glacier, Central Swiss Alps). Geophys. Res. Lett. 2017, 44, 6244-6251. [CrossRef]

8. De Lellis, G.; Ereditato, A.; Niwa, K. Nuclear Emulsions. In Detectors for Particles and Radiation. Part 1: Principles and Methods; Fabjan, C.W., Schopper, H., Eds.; Springer: Berlin/Heidelberg, Germany, 2011; pp. 262-287.

9. Ereditato, A. The Study of Neutrino Oscillations with Emulsion Detectors. Adv. High Energy Phys. 2013, 2013, 382172. [CrossRef]

10. Nakamura, T.; Ariga, A.; Ban, T.; Fukuda, T.; Fukuda, T.; Fujioka, T.; Furukawa, T.; Hamada, K.; Hayashi, H.; Hiramatsu, S.; et al. The \{OPERA\} film: New nuclear emulsion for large-scale, high-precision experiments. Nucl. Instrum. Methods Phys. Res. Sect. A 2006, 556, 80-86. [CrossRef]

11. Kreslo, I.; Cozzi, M.; Ereditato, A.; Hess, M.; Knuesel, J.; Laktineh, I.; Messina, M.; Moser, U.; Pistillo, C.; Pretzl, K. High-speed analysis of nuclear emulsion films with the use of dry objective lenses. JINST 2008, 3, P04006. [CrossRef]

12. Ariga, A.; Ariga, T. Fast $4 \pi$ track reconstruction in nuclear emulsion detectors based on GPU technology. JINST 2014, 9, P04002. [CrossRef]

13. Nagamine, K.; Iwasakia, M.; Shimomura, K.; Ishida, K. Method of probing inner-structure of geophysical substance with the horizontal cosmic-ray muons and possible application to volcanic eruption prediction. Nucl. Instrum. Methods Phys. Res. Sect. A 1995, 356, 585-595. [CrossRef]

14. Procureur, S. Muon imaging: Principles technologies and applications. Nucl. Instrum. Methods Phys. Res. Sect. A 2018, 872, 169-179. [CrossRef]

15. Reyna, D. A Simple Parameterization of the Cosmic-Ray Muon Momentum Spectra at the Surface as a Function of Zenith Angle. arXiv 2006, arXiv:hep-ph/0604145. [CrossRef]

16. Lesparre, N.; Gibert, D.; Marteau, J.; Déclais, Y.; Carbone, D.; Galichet, E. Geophysical muon imaging: Feasibility and limits. Geophys. J. Int. 2010, 183, 1348-1361. [CrossRef]

17. Hebbeker, T.; Timmermans, C. A compilation of high energy atmospheric muon data at sea level. Astropart. Phys. 2002, 18, 107-127. [CrossRef]

18. Nishiyama, R.; Taketa, A.; Miyamoto, S.; Kasahara, K. Monte Carlo simulation for background study of geophysical inspection with cosmic-ray muons. Geophys. J. Int. 2016, 206, 1039-1050. [CrossRef]

19. Groom, D.E.; Mokhov, N.V.; Striganov, S.I. Muon Stopping Power and Range Tables $10 \mathrm{MeV}-100 \mathrm{TeV}$. At. Data Nucl. Data Tables 2001, 78, 183-356. [CrossRef]

20. Nishio, A.; Morishima, K.; Kuwabara, K.; Nakamura, M. Development of nuclear emulsion detector for muon radiography. Phys. Procedia 2015, 80, 74-77. [CrossRef]

21. Aghion, S.; Ahlén, O.; Amsler, C.; Ariga, A.; Ariga, T.; Belov, A.S.; Bonomi, G.; Braünig, P.; Bremer, J.; Brusa, R.S.; et al. Prospects for measuring the gravitational free-fall of antihydrogen with emulsion detectors. JINST 2013, 8, P08013. [CrossRef]

22. Huss, M. Density assumptions for converting geodetic glacier volume change to mass change. Cryosphere 2013, 7, 877-887. [CrossRef] 
23. Jourde, K.; Gibert, D.; Marteau, J.; De Bremond d'Ars, J.; Gardien, S.; Girerd, C.; Ianigro, J.-C.; Carbone, D. Experimental detection of upward going cosmic particles and consequences for correction of density radiography of volcanoes. Geophys. Res. Lett. 2013, 40, 6334-6339. [CrossRef]

24. Tioukov, V.; Kreslo, I.; Petukhov, Y.; Sirri, G. The FEDRA—Framework for emulsion data reconstruction and analysis in the OPERA experiment. Nucl. Instrum. Methods Phys. Res. Sect. A 2006, 559, 103-105. [CrossRef]

(C) 2018 by the authors. Licensee MDPI, Basel, Switzerland. This article is an open access article distributed under the terms and conditions of the Creative Commons Attribution (CC BY) license (http:/ / creativecommons.org/licenses/by/4.0/). 